\title{
Quasi-Periodic Oscillations in magnetars: linking variability and emission
}

\author{
Caroline D'Angelo \\ Instituut Anton Pannekoek, University of Amsterdam, Amsterdam, Netherlands 1098 XH \\ email: c.r.dangelo@uva.nl
}

\begin{abstract}
I present recent results studying flare emission in magnetars. Strong quasi-periodic oscillations observed in the tail of giant magnetar flares are frequently interpreted as evidence for global seismic oscillations. I demonstrate that such a global oscillation is not directly observable in the lightcurve. New work suggests the amplitude for the strongest QPO stays nearly constant in the rotation phases where it is observed, which I argue suggests it is produced by an additional emission process from the star.
\end{abstract}

Keywords. dense matter, radiation mechanisms: general, magnetic fields (magnetohydrodynamics:) MHD, stars: neutron, stars: oscillations, X-rays: stars

\section{Introduction}

In the past few years, considerably theoretical interest has been focused on the seismology of neutron stars (e.g. Levin 2006; Glampedakis et al. 2006; Gabler et al. 2011), largely been motivated by the observation of a series of quasi-periodic oscillations (QPOs) in the decay tails of the giant flares in magnetars SGR 1900+14 and SGR 1806-20 (Israel et al. 2005; Watts \& Strohmayer 2006; Strohmayer \& Watts 2006). The large amount of energy released during a flare $\left(\sim 10^{46} \mathrm{erg}\right)$ is likely powered by a global reconfiguration of the star's magnetic field (Thompson \& Duncan 1995) and some of that energy will trigger large-scale quakes in the star (Duncan 1998).

Comparatively little work has been done to connect the putative starquakes directly to the observed QPOs. Timokhin et al. (2008) recently proposed that the oscillations could be attributed to a variable current density in the stellar magnetosphere, created by twisted magnetic field lines anchored in the vibrating star. These electrons then Compton upscatter photons from the surface of the star, modifying the observed spectrum.

In this article I revisit the properties of the QPOs observed in SGR 1806-20 and argue that correlations (or lack thereof) between the variability on different timescales can be used to put strong constraints not only on the QPO origins but also on the emission mechanisms powering the flares themselves.

\section{Quasi-Periodic Oscillations in SGR 1806-20}

Six distinct QPOs were detected in the SGR 1806-20 giant flare using X-ray data from both the RHESSI and RXTE satellites. The QPOs have central frequencies between 17 and $1837 \mathrm{~Hz}$, with fractional rms amplitudes between 4 and $20 \%$. They are further characterized by a high degree of coherence: of the six detected QPOs, only the one at $150 \mathrm{~Hz}$ has a width (full-width at half maximum) of $17 \mathrm{~Hz}$; the others have widths between 1 and $5 \mathrm{~Hz}$ (Strohmayer \& Watts 2006). There is some evidence for energy dependence in the QPO at $625 \mathrm{~Hz}$, which had an rms amplitude of $\sim 8 \%$ below $100 \mathrm{keV}$, but of rms $\sim 20 \%$ between 100-200keV (Watts \& Strohmayer 2006). The energy dependence of the 
other QPOs is notclearly detected, but cannot be excluded due to uncertainties in the measured photon energies.

Different QPOs were detected at different time intervals in the decay tail of the flare and at different phases in the rotational pulse profile. The majority of the QPOs were strongest beginning $\sim 200$ s after the main flare, and in the 'interpulse' region in phase, where the flux in the lightcurve is at a minimum (see fig. 1 for the the averaged pulse profile). The strongest QPO in the RXTE data has a central frequency of $93 \mathrm{~Hz}$ and fractional rms amplitude of $\sim 20 \%$. It is also detectable over a significant portion of the rotation phase and hence can be used to study the relationship between the QPOs and the pulse profile, as well as relationships between the QPO and broadband noise.

\section{Direct detection of a starquake}

D'Angelo \& Watts (2012) studied whether a starquake could have an observable effect directly on the lightcurve itself by shaking the emitting region. If the pulse profile is very steep (i.e. some component of the pulse is beamed) then the sharp edge of the beam will amplify the underlying motion of the surface, much like a flashlight wiggling in and out of an observer's line of sight. The change to the rotational phase from the crust motion is given by:

$$
\Delta \Phi=\frac{\Delta x}{R_{*} \sin i \sin \alpha} \sin \left(2 \pi \nu_{0} t\right),
$$

where $\frac{\Delta x}{R_{*}}$ is the fractional amplitude of the starquake, $\nu_{0}$ is its frequency, and $\sin i$ and $\sin \alpha$ are geometrical factors depending on the beam orientation.

D'Angelo \& Watts (2012) found that although significant amplification of a star quake is possible, for the observed lightcurves and realistic, the effect can be excluded. The fractional rms amplitude of a QPO with phase change $\Delta \Phi$ is given by:

$$
A \sim \frac{\frac{d P}{d \Phi} \Delta \Phi}{\langle P \Phi\rangle},
$$

where $P(\Phi)$ is the pulse profile as a function of rotation phase. The amplification provided by a steep gradient is not enough to make a starquake (with $\Delta x / R_{*}<0.01$ ) directly detectable. This result also excludes the possibility that weak, extremely steep 'pencil beams' (unresolved in the lightcurve) can provide the amplification. The amplification factor in that case will be given by eq. 3.2 times an additional factor $P_{\text {beam }} /\langle P\rangle$, the fractional amplitude of the steep beam. The beam gradients required in this case are steep enough to be plausibly excluded.

This result strongly suggests the QPOs are produced by variations in the amplitude of the emission itself, rather than the starquake directly.

\section{Modulation versus Emission}

The physical properties of the QPO can be somewhat constrained from the observed variability of the lightcurve. This is most easily seen from the power spectrum, essentially the squared amplitude of the Fourier transform of a segment of the lightcurve (e.g. van der Klis 1989). The left and right panels of figure 1 show power spectra from the pulsed tail of the giant flare, centered at two different phases of the pulse profile (shown in the bottom panel). In each power spectrum the QPO at $93 \mathrm{~Hz}$ is clearly visible, and a fit to the QPO is overlaid. The QPO is significantly narrower in the interpulse region, and the broadband noise (below $\sim 100 \mathrm{~Hz}$ ) is lower (a second QPO is seen at $30 \mathrm{~Hz}$ in the 

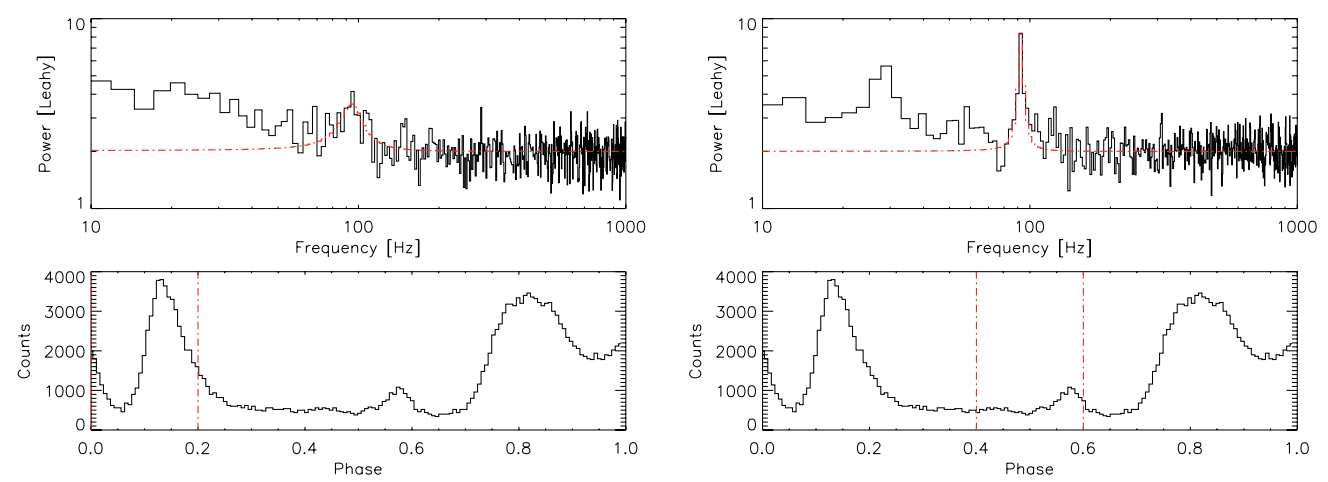

Figure 1. Power spectrum and pulse profile of the tail of the SGR 1806-20 giant flare for two different segments of the rotational phase (shown by the vertical lines). The QPO at $93 \mathrm{~Hz}$ is fit with a Lorentzian distribution (overlaid in red dash-dotted line).

interpulse spectrum). At the same time, the mean flux in the lightcurve is $\sim 60 \%$ lower than in the secondary pulse.

There are two obvious ways that the observed intensity can vary at the QPO frequencies. Either the surface flux can be modulated by a quasi-periodically varying process (like changing optical depth to electron scattering, cf. Timohkin et al. 2008), or the amount of flux being emitted by the star can vary, either through variations in the overall surface emission or via some other instability that produces emission. At present the idea of variable emission in the magnetar magnetosphere is purely speculative, but mechanisms for producing QPOs in solar flares are an active research topic, and some of these could potentially be relevant for magnetar flares as well (see e.g. the review by Nakariakov \& Melnikov 2009).

The difference between an emitting process and a modulating one should be observable in the phase-resolved QPOs. A modulating process should produce a correlation between the absolute amplitude of the QPO and the mean flux. In contrast, an emission process should stay constant in phase, and be stronger at phases when the mean flux is lower. Figure 2 shows the fractional change in flux as a function of phase (black line solid), overplotted with the fractional change in QPO amplitude (integrated power over $20 \mathrm{~Hz}$ centered at $93 \mathrm{~Hz}$; red dot-dashed line). As is evident from the figure, the QPO amplitude does not drop as much in the interpulse region, then disappears altogether at the main peak of the burst. The lack of correlation between mean flux and QPO amplitude would suggest that an additional emission process is responsible for its production.

\section{Markov Chain Monte Carlo Simulations}

Determining the amplitude of the QPOs - particularly those below $100 \mathrm{~Hz}$ - is complicated by the presence of low-frequency broadband noise (visible in the left panel of fig. 1). Part of the signal at $93 \mathrm{~Hz}$ could originate from red noise and not the QPO process, but disentangling the two components is not straightforward (see e.g. Vaughan 2005).

To quantify the uncertainty in QPO amplitde, we use Markov Chain Monte Carlo simulations to generate a series of realizations of a power spectrum with a broadband component and a QPO given by the best fit to the observed spectrum (Vaughan 2010). The variation in the resulting measured parameters can be used to constrain the uncertainty on the QPO fit, and more accurately determine the variation in QPO amplitude as a function of phase. Preliminary results of this analysis suggest that the amplitude observed QPO at $93 \mathrm{~Hz}$ is consistent with remaining constant over the rotation phase 


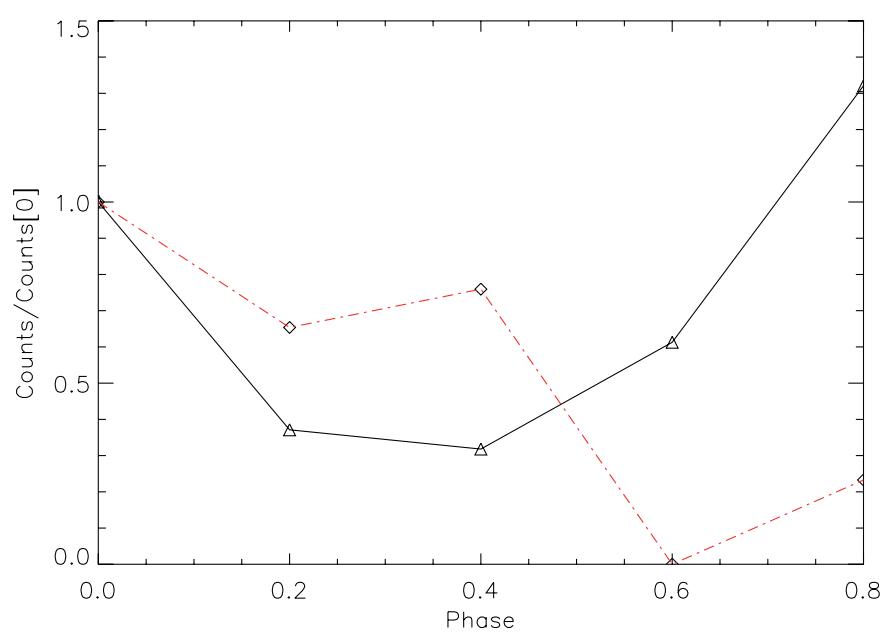

Figure 2. Mean flux (black solid line) and $93 \mathrm{~Hz}$ QPO amplitude (red dash-dotted line) as a function of phase, for the tail of the SGR 1806-20 giant flare. For the phases where the QPO is detected, the amplitude is consistent with being constant while the mean flux varies substantially.

where it is detectable. This would seem to suggest it is independent of the secondary pulse peak, and point to an underlying emission process. The definitive results will be published in a forthcoming paper.

\section{Conclusions}

The variability of magnetar giant flares on different timescales shows correlations that can constrain the underlying emission mechanism, both for the QPOs and potentially the emission from the giant flares themselves. We have excluded the possibility of directly detecting surface oscillation of the magnetar crust, and have presented preliminary evidence that suggests an additional emission mechanism might be active to produce the quasi-periodic oscillations.

\section{References}

D'Angelo, C. R. \& Watts, A. L. 2012, ApJL, 751, L41

Duncan, R. C. 1998, ApJL, 498, L45

Gabler, M., Cerdá Durán, P., Font, J. A., Müller, E., \& Stergioulas, N. 2011, MNRAS, 410, L37

Glampedakis, K., Samuelsson, L., \& Andersson, N. 2006, MNRAS, 371, L74

Israel, G. L., Belloni, T., Stella, L., et al. 2005, ApJL, 628, L53

Levin, Y. 2006, MNRAS, 368, L35

Nakariakov, V. M. \& Melnikov, V. F. 2009, Space Sci. Rev., 149, 119

-. 2006, ApJ, 653, 593

Thompson, C. \& Duncan, R. C. 1995, MNRAS, 275, 255

Timokhin, A. N., Eichler, D., \& Lyubarsky, Y. 2008, ApJ, 680, 1398

van der Klis, M. 1989, in Timing Neutron Stars, ed. H. Ögelman \& E. P. J. van den Heuvel, 27

Vaughan, S. 2005, A\& $A, 431,391$

-. 2010, MNRAS, 402, 307

Watts, A. L. \& Strohmayer, T. E. 2006, ApJL, 637, L117 